\title{
Toxic and essential trace elements in adolescents
}

\author{
Ebba Bárány
}

There is a need for knowledge of trace element levels in different population groups. Several applications can be identified. One is as reference levels that can be used to detect changes in environmental exposure. Another is to reveal deficiency of essential elements, or in some cases toxicity. Toxic elements need to be monitored, as the exposure may be close to levels where adverse effects can arise. Absorption of elements occurs via the gastrointestinal tract and the lungs, and the absorbed fraction may depend on the route of exposure.

Diet is the main source of exposure to trace elements in the general population that are not occupationally exposed. Toxic elements may enter the food chain through contamination of the environment, e.g. through atmospheric deposition or naturally high levels in soil. Essential elements are widespread in most foods, as they are also essential to plants and animals used for food production.

Adolescents constitute a little studied group in regard to trace element levels in blood. They have a high recommended energy and nutrient intake per kg body weight. Moreover, a high energy intake may expose them to more toxic trace elements via the diet than adults. However, many adolescents do not consume an adequate diet and may therefore risk deficiency of essential elements. In a longitudinal Swedish nutritional survey, almost 400 adolescents (boys and girls) were sampled for blood and serum at the ages of 15 and 17 years for a trace element study within the survey. The adolescents were living in the two Swedish cities, Uppsala and Trollhättan. The cities differ in that Trollhättan has long had metal industries such as several smelters, while Uppsala is a university city, with almost no industrial metal emissions. The two cities also represent different socioeconomic structures, with a higher percentage of the adolescents' parents having university-level education in Uppsala.
The elements analysed were the well-known toxic elements cadmium, mercury and lead, as well as the essential elements cobalt, copper, zinc and selenium (1). The elements rubidium, platinum, palladium, rhodium, thallium and tungsten were also analysed. The analytical method used was inductively coupled plasma mass spectrometry (ICP-MS), which enables rapid and simultaneous multielement analyses. The trace element concentrations were then related to the adolescents' age, gender, socioeconomic status and residential area (city) (2). It should be noted that the age factor may also reflect temporal changes in environmental exposure. This is mainly relevant for toxic elements. The influence of fish consumption on mercury and selenium concentrations, and of dental amalgam fillings on mercury concentrations, was also investigated (3).

The adolescents' blood concentration of lead was low from an international perspective, and also decreased between 15 and 17 years of age (2, 4). Decreasing blood lead has been reported in conjunction with decreasing use of leaded petrol, but the present decrease may also be a result of dilution of body burden in the growing adolescents. There was also a significant difference in blood lead concentration between Uppsala and Trollhättan ( $p<0.0005$, Fig. 1). Boys had higher levels of lead in the blood than did girls. Reasons for a gender difference may be a higher haematocrit, or a higher energy intake in the boys. The levels of cadmium in blood were significantly higher in smokers than in non-smokers $(p<$ $0.0005)$. Blood cadmium was also positively influenced by socioeconomic status (2). The reason for this relationship is not obvious, but the higher consumption of vegetables in the higher socioeconomic groups may play a role, as vegetables are one of the main food groups contributing cadmium to the diet.

It was shown that in this study population, fish consumption was a statistically significant predic- 


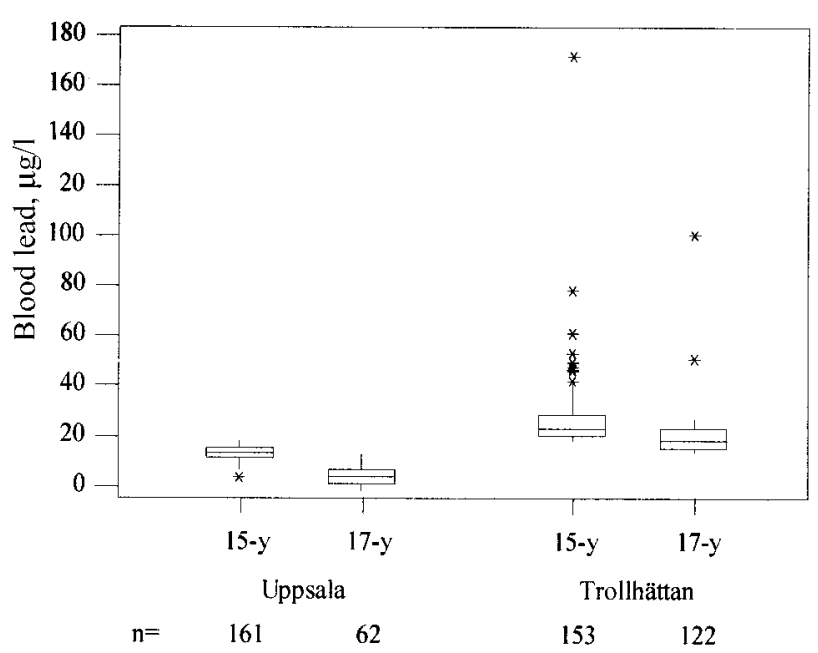

Fig. 1. Blood lead $\left(\mu \mathrm{g}^{-1}\right)$ in adolescents from the Swedish cities Uppsala and Trollhättan at the ages of 15 (15-y) and 17 years $(17-y)$. The box is defined by the upper and lower quartiles, with the median marked by a subdivision of the box. The whiskers are the lines that extend from the top and bottom of the boxes to the lowest and highest observations, excluding outliers (circles) with values that are more than 1.5 box-length s from the median.

tor of mercury in both blood and serum (3), even though the adolescents consumed only a few fish meals per month. However, the concentrations of selenium in blood and serum were not influenced by fish consumption. The highest selenium concentrations were found in boys from Uppsala, and the lowest in girls from Trollhättan (2). The number of dental amalgam fillings significantly influenced the mercury concentration in serum (3).

Essential trace elements are regulated by homoeostatic mechanisms in the body, and it is important to take this into account when interpreting analytical data. The copper levels increased with age in the girls, probably because of hormonal changes during puberty $(p<0.006$, Fig. 2$)$. The concentration of serum copper was also elevated in 17-year old smoking girls compared with non-smokers $(p<$ 0.0005 , Fig. 2). The cobalt concentration was higher in girls, while boys had higher levels of zinc in serum. None of the essential elements was influenced by socioeconomic status (4).

Residential area significantly influenced each of the 13 analysed elements (4). The differences in element levels, however, were in different directions, e.g. blood lead levels were higher in Trollhättan, while serum mercury was higher in Uppsala. Therefore, there was no clear evidence that the higher metal emissions in Trollhättan caused these differences.

This study demonstrates the impact of several factors on trace element levels in adolescents, thus

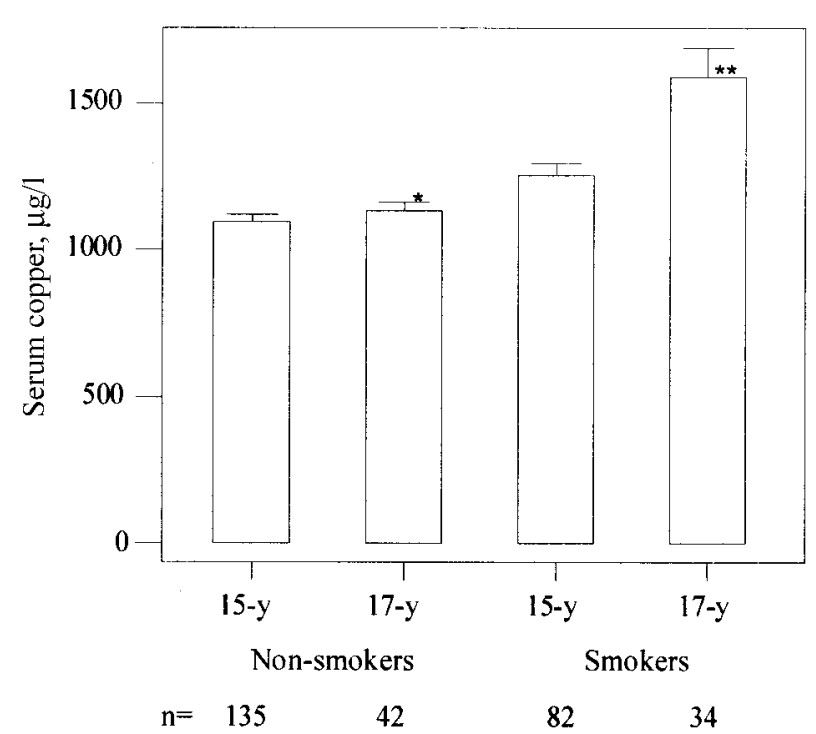

Fig. 2. Serum copper $\left(\mu \mathrm{g}^{-1}\right)$ in girls at the ages of $15(15-\mathrm{y})$ and 17 years (17-y). The bars represent mean and standard error. ${ }^{*} p<0.006$ compared with non-smoking 15 -year-olds, ${ }^{* *} p<0.0005$ compared with non-smoking 15 - and 17 -year-olds, and with smoking 15-year-olds.

showing the relevance of reporting the subjects' background in detail in trace element studies.

\section{References}

1. Bárány E, Bergdahl IA, Schütz A, Skerfving S, Oskarsson A: Inductively coupled plasma mass spectrometry for direct multi-element analysis of diluted human blood and serum. $J$ Anal At Spectrom 1997:12:1005-9.

2. Bárány E, Bergdahl IA, Bratteby L-E, Lundh T, Samuelson G, Schütz A, Skerfving $S$, Oskarsson A: Trace elements in Swedish adolescents: influence of age, gender, residential area and socioeconomic status. Environ Res (in press).

3. Bárány E, Bergdahl IA, Bratteby L-E, Lundh T, Samuelson G, Schütz A, Skerfving S, Oskarsson A: Mercury and selenium in whole blood and serum in relation to fish consumption and amalgam fillings in adolescents. Submitted.

4. Bárány E, Bergdahl IA, Bratteby L-E, Lundh T, Samuelson G, Skerfving S, Oskarsson A: Trace element levels in whole blood and serum from Swedish adolescents. Sci Tot Environ 2002:286:129-41.

The article is based on the author's thesis, which was conducted at the Department of Pharmacology and Toxicology, Swedish University of Agricultural Sciences, Uppsala, Sweden. Defended on February 22, 2002. ISBN: 91-576-6354-8

\section{Ebba Bárány}

Department of Pharmacology and Toxicology, Swedish

University of Agricultural Sciences, BMC, Box 573, SE-75 I 23

Uppsala, Sweden. E-mail: Ebba.Barany@farmtox.slu.se 